\title{
Utility of Syndromic Surveillance Using Novel Clinical Data Sources
}

\author{
Rebecca Zwickl*, Charles Ishikawa and Laura C. Streichert \\ ISDS, Brighton, MA, USA
}

\section{Objective}

To document the current evidence base for the use of electronic health record (EHR) data for syndromic surveillance using emergency department, urgent care clinic, hospital inpatient, and ambulatory clinical care data.

\section{Introduction}

Historically, syndromic surveillance has primarily involved the use of near real-time data sent from hospital emergency department (EDs) and urgent care (UC) clinics to public health agencies. The use of data from inpatient and ambulatory settings is now gaining interest and support throughout the United States, largely as a result of the Stage 2 and 3 Meaningful Use regulations [1]. Questions regarding the feasibility and utility of applying a syndromic approach to these data sources are hampering the development of systems to collect, analyze, and share this potentially valuable information. Solidifying the evidence base and communicating the results to the public health surveillance community may help to initiate and build support for using these data to advance surveillance functions.

\section{Methods}

We conducted a literature search in the published and grey literature that scanned for relevant articles in the Google Scholar, Pub Med, and EBSCO Information Services databases. Search terms included: "inpatient/ambulatory electronic health record"; "ambulatory/inpatient/hospital/outpatient/chronic disease syndromic surveillance"; and "EHR syndromic surveillance". Information gleaned from each article included data use, data elements extracted, and data quality indicators. In addition, several stakeholders who provided input on the September 2012 ISDS Recommendations [2] also provided articles that were incorporated into the literature review.

ISDS also invited speakers from existing inpatient and ambulatory syndromic surveillance systems to give webinar presentations on how they are using data from these novel sources.

\section{Results}

The number of public health agencies (PHAs) routinely receiving ambulatory and inpatient syndromic surveillance data is substantially smaller than the number receiving ED and UC data. Some health departments, private medical organizations (including HMOs), and researchers are conducting syndromic surveillance and related research with health data captured in these clinical settings [2].

In inpatient settings, many of the necessary infrastructure and analytic tools are already in place. Syndromic surveillance with inpatient data has been used for a range of innovative uses, from monitoring trends in myocardial infarction in association with risk factors for cardiovascular disease [3] to tracking changes in incident-related hospitalizations following the 2011 Joplin, Missouri tornado [3].
In contrast, ambulatory systems face a need for new infrastructure, as well as pose a data volume challenge. The existing systems vary in how they address data volume and what types of encounters they capture. Ambulatory data has been used for a variety of uses, from monitoring gastrointestinal infectious disease [3], to monitoring behavioral health trends in a population, while protecting personal identities [4].

\section{Conclusions}

The existing syndromic surveillance systems and substantial research in the area indicate an interest in the public health community in using hospital inpatient and ambulatory clinical care data in new and innovative ways. However, before inpatient and ambulatory syndromic surveillance systems can be effectively utilized on a large scale, the gaps in knowledge and the barriers to system development must be addressed. Though the potential use cases are well documented, the generalizability to other settings requires additional research, workforce development, and investment.

\section{Keywords}

Syndromic surveillance; EHR; Meaningful Use

\section{Acknowledgments}

We thank the ISDS Meaningful Use Workgroup for their assistance with the literature review, and all the presenters in the ISDS Meaningful Use Webinar series (http://www.syndromic.org/webinars/meaningfuluse). Work supported by CDC through ISDS contract with Task Force for Global Health.

\section{References}

1. Health Information Technology for Economic and Clinical Health (HITECH) Act. Title XIII of Division A and Title IV of Division B of the American Recovery and Reinvestment Act of 2009 2009; Pub. L. No. 111-5.

2. ISDS. Electronic Syndromic Surveillance Using Hospital Inpatient and Ambulatory Clinical Care Electronic Health Record Data: Recommendations from the ISDS Meaningful Use Workgroup. 2012. www.syndromic.org

3. Various presenters. ISDS Meaningful Use Webinar Series: 3/13/21/2012. http://www.syndromic.org/webinars/meaningfuluse

4. Pavlin JA, Murdock P, Elbert E, Milliken C, Hakre S. Conducting population behavioral health surveillance by using automated diagnostic and pharmacy data systems. MMWR 2004;53 (Supp.):166-172.

\section{*Rebecca Zwick}

E-mail: bzwickl@syndromic.org 